\title{
GENERALIZED QUATERNIONIC SCHUR FUNCTIONS IN THE BALL AND HALF-SPACE AND KREIN-LANGER FACTORIZATION
}

\author{
DANIEL ALPAY, FABRIZIO COLOMBO, AND IRENE SABADINI
}

\begin{abstract}
In this paper we prove a new version of Krein-Langer factorization theorem in the slice hyperholomorphic setting which is more general than the one proved in [8]. We treat both the case of functions with $\kappa$ negative squares defined on subsets of the quaternionic unit ball or on subsets of the half space of quaternions with positive real part. A crucial tool in the proof of our results is the Schauder-Tychonoff theorem and an invariant subspace theorem for contractions in a Pontryagin space.
\end{abstract}

\section{INTRODUCTION}

1.1. Some history. Functions analytic and contractive in the open unit disk $\mathbb{D}$ play an important role in various fields of mathematics, in electrical engineering and digital signal processing. They bear various names, and in particular are called Schur functions. We refer to [32] for reprints of some of the original works. André Bloch's 1926 memoir [19] contains also valuable historical background.

Schur functions admit a number of generalizations, within function theory of one complex variable and outside. A $\mathbb{C}^{r \times s}$-valued function $S$ analytic in $\mathbb{D}$ is a Schur function if and only if the kernel

$$
K_{S}(z, w)=\frac{I_{r}-S(z) S(w)^{*}}{1-z \bar{w}}
$$

is positive definite in $\mathbb{D}$. In fact, much more is true. It is enough to assume that the kernel $K_{S}(z, w)$ is positive definite in some subset $\Omega$ of $\mathbb{D}$ to insure that $S$ is the restriction to $\Omega$ of a (not necessarily unique) function analytic and contractive in $\mathbb{D}$; see [2, 28].

A Schur function has no poles inside the open unit disk. Motivated by lowering a lower bound given by Carathéodory and Fejér in an interpolation

1991 Mathematics Subject Classification. 47A48, 30G35, 30D50.

Key words and phrases. Schur functions, realization, reproducing kernels, slice hyperholomorphic functions, Blaschke products.

D. Alpay thanks the Earl Katz family for endowing the chair which supported his research, and the Binational Science Foundation Grant number 2010117. I. Sabadini was partially supported by the FIRB Project Geometria Differenziale Complessa e Dinamica Olomorfa. 
problem, Takagi considered in [46, 47] rational functions bounded by 1 in modulus on the unit circle and with poles inside $\mathbb{D}$. These are the first instances of what is called a generalized Schur function. Later studies of such functions include Chamfy [22], Dufresnoy [31] (these authors being motivated by the study of Pisot numbers), Delsarte, Genin and Kamp [25, 27. and Krein and Langer [36, 37, 38, to mention a few names. The precise definition of a generalized Schur function was given (in the setting of operator valued functions) by Krein and Langer [36]:

Definition 1.1. A $\mathbb{C}^{r \times s}$-valued function analytic in an open subset $\Omega$ of the unit disc is called a generalized Schur function if the kernel $K_{S}$ has a finite number (say $\kappa$ ) of negative squares in $\Omega$, meaning that for every choice of $N \in \mathbb{N}, c_{1}, \ldots, c_{N} \in \mathbb{C}^{r}$ and $w_{1}, \ldots, w_{N} \in \Omega$, the $N \times N$ Hermitian matrix with $(\ell, j)$ entry $c_{\ell}^{*} K_{S}\left(w_{\ell}, w_{j}\right) c_{j}$ has at most $\kappa$ strictly negative eigenvalues, and exactly $\kappa$ strictly negative eigenvalues for some choice of $N, c_{1}, \ldots, c_{N}, w_{1}, \ldots, w_{N}$.

In the setting of matrix-valued functions, the result of Krein and Langer states that $S$ is a generalized Schur function if and only if it is the restriction to $\Omega$ of a function of the form

$$
B_{0}(z)^{-1} S_{0}(z),
$$

where $S_{0}$ is a $\mathbb{C}^{r \times s}$-valued Schur function and $B_{0}$ is $\mathbb{C}^{r \times r}$-valued Blaschke product of degree $\kappa$. Besides [36], there exist various proofs of this result; see for instance [9, p. 141], 21].

That one cannot remove the analyticity condition in the result of Krein and Langer when $\kappa>0$ is illustrated by the well known counterexample $S(z)=\delta_{0}(z)$, where $\delta_{0}(z)=0$ if $z \neq 0$ and $\delta_{0}(0)=1$ (see for instance [9, p. 82]). Taking into account that $z^{n} \delta_{0}(z) \equiv 0$ for $n>0$ we have

$$
\begin{aligned}
\frac{1-S(z) S(w)^{*}}{1-z \bar{w}} & =\frac{1}{1-z \bar{w}}-\sum_{n=0}^{\infty} z^{n} \delta_{0}(z) \bar{w}^{n} \delta_{0}(w) \\
& =\frac{1}{1-z \bar{w}}-\delta_{0}(z) \delta_{0}(w) .
\end{aligned}
$$

The reproducing kernel Hilbert space associated with $\delta_{0}(z) \delta_{0}(w)$ is $\mathbb{C} \delta_{0}$ and has a zero intersection with $\mathbf{H}_{2}(\mathbb{D})$, and hence the kernel $K_{S}$ has one negative square.

1.2. The slice hyperholomorphic case. Schur functions have been extended to numerous settings, and we mention in particular the setting of several complex variables [1, 18], compact Riemann surfaces [15] and hypercomplex functions [12, 13. Generalized Schur functions do not exist necessarily in all these settings.

In [6] we began a study of Schur analysis in the framework of slice hyperholomorphic functions. The purpose of this paper is to prove the theorem of Krein and Langer (we considered a particular case in [8]) and we treat 
both the unit ball and half-space cases in the quaternionic setting. To that purpose we need in particular the following:

(i) The notion of negative squares and of reproducing kernel Pontryagin spaces in the quaternionic setting. This was done in [14.

(ii) The notion of generalized Schur functions and of Blaschke products, see [7].

(iii) A result on invariant subspaces of contractions in quaternionic Pontryagin spaces.

(iv) The notion of realization in the slice-hyperholomorphic setting, in particular when the state space is a one-sided (as opposed to twosided) Pontryagin space.

The paper contains 6 sections, besides the Introduction. Section 2 contains a quick survey of the Krein-Langer result in the classical case. Section 3 introduces slice hyperholomorphic functions and discusses Blaschke products. Section 4 contains some useful results in quaternionic functional analysis, among which Schauder-Tychonoff theorem. In Section 5 we present generalized Schur functions and their realizations. Finally, in Section 6 we prove the Krein-Langer factorization for generalized Schur functions defined in a subset of the unit ball and finally, in Section 7, we state the analogous result in the case of the half-space.

\section{A SURVEy of the Classical CASE}

The celebrated one-to-one correspondence between positive definite functions and reproducing kernel Hilbert spaces (see [16]) extends to the indefinite case, when one considers functions with a finite number of negative squares and reproducing kernel Pontryagin spaces; see [10, 43, 44]. We recall the definition of the latter for the convenience of the reader.

A complex vector space $\mathcal{V}$ endowed with a sesquilinear form $[\cdot, \cdot]$ is called an indefinite inner product space (which we will also denote by the pair $(\mathcal{V},[\cdot, \cdot]))$. The form $[\cdot, \cdot]$ defines an orthogonality: two vectors $v, w \in \mathcal{V}$ are orthogonal if $[v, w]=0$, and two linear subspaces $\mathcal{V}_{1}$ and $\mathcal{V}_{2}$ of $\mathcal{V}$ are orthogonal if every vector of $\mathcal{V}_{1}$ is orthogonal to every vector of $\mathcal{V}_{2}$. Orthogonal sums will be denoted by the symbol $[+]$. Note that two orthogonal spaces may intersect. We will denote by the symbol $[\oplus]$ a direct orthogonal sum. A complex vector space $\mathcal{V}$ is a Krein space if it can be written (in general in a non-unique way) as

$$
\mathcal{V}=\mathcal{V}_{+}[\oplus] \mathcal{V}_{-}
$$

where $\left(\mathcal{V}_{+},[\cdot, \cdot]\right)$ and $\left(\mathcal{V}_{-},-[\cdot, \cdot]\right)$ are Hilbert spaces. When the space $\mathcal{V}_{-}$ (or, as in [35, the space $\mathcal{V}_{+}$) is finite dimensional (note that this property does not depend on the decomposition), $\mathcal{V}$ is called a Pontryagin space. The space $\mathcal{V}$ endowed with the form

$$
\langle h, g\rangle=\left[h_{+}, g_{+}\right]-\left[h_{-}, g_{-}\right]
$$


where $h=h_{+}+h_{-}$and $g=g_{+}+g_{-}$are the decompositions of $f, g \in \mathcal{V}$ along (2.1), is a Hilbert space. One endows $\mathcal{V}$ with the corresponding topology. This topology is independent of the decomposition (2.1) (the latter is not unique, but is in the definite case).

Let now $T$ be a linear densely defined map from a Pontryagin space $\left(\mathcal{P}_{1},[\cdot, \cdot]_{1}\right)$ into a Pontryagin space $\left(\mathcal{P}_{2},[\cdot, \cdot]_{2}\right)$. Its adjoint is the operator $T^{*}$ with domain $\operatorname{Dom}\left(T^{*}\right)$ defined by:

$$
\left\{g \in \mathcal{P}_{2}: h \mapsto[T h, g]_{2} \text { is continuous }\right\} \text {. }
$$

One then defines by $T^{*} g$ the unique element in $\mathcal{P}_{1}$ which satisfies

$$
[T h, g]_{2}=\left[h, T^{*} g\right]_{1} \text {. }
$$

Such an element exists by the Riesz representation theorem.

The operator $T$ is called a contraction if

$$
[T h, T h]_{2} \leq[h, h]_{1}, \quad \forall h \in \operatorname{Dom}(T),
$$

while it is said to be a coisometry if $T T^{*}=I$.

Theorem 2.1. A densely defined contraction between Pontryagin spaces of the same index has a unique contractive extension and its adjoint is also a contraction.

We refer to [17, 20, 30] for the theory of Pontryagin and Krein spaces, and of their operators.

With these definitions, we can state the following theorem, which gathers the main properties of generalized Schur functions.

Theorem 2.2. Let $S$ be a $\mathbb{C}^{r \times s}$-valued function analytic in a neighborhood $\Omega$ of the origin. Then the following are equivalent:

(1) The kernel $K_{S}(z, w)$ has a finite number of negative squares in $\Omega$.

(2) There is a Pontryagin space $\mathcal{P}$ and a coisometric operator matrix

$$
\left(\begin{array}{ll}
A & B \\
C & D
\end{array}\right): \mathcal{P} \oplus \mathbb{C}^{s} \rightarrow \mathcal{P} \oplus \mathbb{C}^{r}
$$

such that

$$
S(z)=D+z C(I-z A)^{-1} B, \quad z \in \Omega .
$$

(3) There exists a $\mathbb{C}^{r \times s}$-valued Schur function $S_{0}$ and $a \mathbb{C}^{r \times r}$-valued Blaschke product $B_{0}$ such that

$$
S(z)=B_{0}(z)^{-1} S_{0}(z), \quad z \in \Omega .
$$

As a corollary we note that $S$ can be extended to a function of bounded type in $\mathbb{D}$, with boundary limits almost everywhere of norm less than or equal 1.

We note the following:

(a) When the pair $(C, A)$ is observable, meaning

$$
\cap_{n=0}^{\infty} \operatorname{ker} C A^{n}=\{0\},
$$


the realization (2.2) is unique, up to an isomorphism of Pontryagin spaces. (b) One can take for $\mathcal{P}$ the reproducing kernel Pontryagin space $\mathcal{P}(S)$ with reproducing kernel $K_{S}$. When $0 \in \Omega$ we have the backward shift realization

$$
\begin{aligned}
A f & =R_{0} f, \\
B c & =R_{0} S c, \\
C f & =f(0), \\
D c & =S(0) c,
\end{aligned}
$$

where $f \in \mathcal{P}(S), c \in \mathbb{C}^{s}$ and where $R_{0}$ denotes the backward shift operator

$$
R_{0} f(z)=\left\{\begin{array}{l}
\frac{f(z)-f(0)}{z}, z \neq 0, \\
f^{\prime}(0), \quad z=0 .
\end{array}\right.
$$

See [9] for more details on this construction, and on the related isometric and unitary realizations.

\section{Slice hyperholomorphic FUnCtions and Blaschke PROdUCtS}

Let $\mathbb{H}$ be the real associative algebra of quaternions, where a quaternion $p$ is denoted by $p=x_{0}+i x_{1}+j x_{2}+k x_{3}, x_{i} \in \mathbb{R}$, and the elements $\{1, i, j, k\}$ satisfy the relations $i^{2}=j^{2}=k^{2}=-1, i j=-j i=k, j k=-k j=i, k i=$ $-i k=j$. As is customary, $\bar{p}=x_{0}-i x_{1}-j x_{2}-k x_{3}$ is called the conjugate of $p$, the real part $x_{0}=\frac{1}{2}(p+\bar{p})$ of a quaternion is also denoted by $\operatorname{Re}(p)$, while $|p|^{2}=p \bar{p}$. The symbol $\mathbb{S}$ denotes the 2 -sphere of purely imaginary unit quaternions, i.e.

$$
\mathbb{S}=\left\{p=i x_{1}+j x_{2}+k x_{3} \mid x_{1}^{2}+x_{2}^{2}+x_{3}^{2}=1\right\} .
$$

If $I \in \mathbb{S}$ then $I^{2}=-1$ and any nonreal quaternion $p=x_{0}+i x_{1}+j x_{2}+k x_{3}$ uniquely determines an element $I_{p}=\left(i x_{1}+j x_{2}+k x_{3}\right) /\left|i x_{1}+j x_{2}+k x_{3}\right| \in \mathbb{S}$. (We note that later $i, j, k$ may also denote some indices, but the context will make clear the use of the notation).

Let $\mathbb{C}_{I}$ be the complex plane $\mathbb{R}+I \mathbb{R}$ passing through 1 and $I$ and let $x+I y$ be an element on $\mathbb{C}_{I}$. Any $p=x+I y$ defines a 2-sphere $[p]=\{x+J y: J \in \mathbb{S}\}$. We now recall the notion of slice hyperholomorphic function:

Definition 3.1. Let $\Omega \subseteq \mathbb{H}$ be an open set and let $f: \Omega \rightarrow \mathbb{H}$ be a real differentiable function. Let $I \in \mathbb{S}$ and let $f_{I}$ be the restriction of $f$ to the complex plane $\mathbb{C}_{I}$. We say that $f$ is a (left) slice hyperholomorphic function in $\Omega$ if, for every $I \in \mathbb{S}, f_{I}$ satisfies

$$
\frac{1}{2}\left(\frac{\partial}{\partial x}+I \frac{\partial}{\partial y}\right) f_{I}(x+I y)=0 .
$$

We say that $f$ is a right slice hyperholomorphic function in $\Omega$ if, for every $I \in \mathbb{S}, f_{I}$ satisfies

$$
\frac{1}{2}\left(\frac{\partial}{\partial x} f_{I}(x+I y)+\frac{\partial}{\partial y} f_{I}(x+I y) I\right)=0 .
$$


The set of slice hyperholomorphic functions on $\Omega$ will be denoted by $\mathcal{R}(\Omega)$. It is a right linear space on $\mathbb{H}$.

Slice hyperholomorphic functions possess good properties when they are defined on the so called axially symmetric slice domains defined below.

Definition 3.2. Let $\Omega$ be a domain in $\mathbb{H}$. We say that $\Omega$ is a slice domain (s-domain for short) if $\Omega \cap \mathbb{R}$ is non empty and if $\Omega \cap \mathbb{C}_{I}$ is a domain in $\mathbb{C}_{I}$ for all $I \in \mathbb{S}$. We say that $\Omega$ is axially symmetric if, for all $q \in \Omega$, the sphere $[q]$ is contained in $\Omega$.

A function $f$ slice hyperholomorphic on an axially symmetric s-domain $\Omega$ is determined by its restriction to any complex plane $\mathbb{C}_{I}$, see [24, Theorem 4.3.2].

Theorem 3.3 (Structure formula). Let $\Omega \subseteq \mathbb{H}$ be an axially symmetric $s$ domain, and let $f \in \mathcal{R}(\Omega)$. Then for any $x+J y \in \Omega$ the following formula holds

$$
f(x+J y)=\frac{1}{2}[f(x+I y)+f(x-I y)+J I(f(x-I y)-f(x+I y))] .
$$

As a consequence of this result, we have the following definition:

Definition 3.4. Let $\Omega$ be an axially symmetric s-domain. Let $h: \Omega \cap \mathbb{C}_{I} \rightarrow$ $\mathbb{H}$ be a holomorphic map. Then it admits a (unique) left slice hyperholomorphic extension $\operatorname{ext}(h): \Omega \rightarrow \mathbb{H}$ defined by:

$$
\operatorname{ext}(h)(x+J y)=\frac{1}{2}[h(x+I y)+h(x-I y)+J I(h(x-I y)-h(x+I y))] .
$$

Remark 3.5. Let $\Omega \subseteq \mathbb{H}$ be an axially symmetric s-domain and let $f, g \in$ $\mathcal{R}(\Omega)$. We can define a suitable product, called the $\star$-product, such that the resulting function $f \star g$ is slice hyperholomorphic. We first define a product between the restrictions $f_{I}, g_{I}$ of $f, g$ to $\Omega \cap \mathbb{C}_{I}$. This product can be extended to the whole $\Omega$ using formula (3.2). Outside the spheres associated with the zeroes of $f \in \mathcal{R}(\Omega)$ we can consider its slice regular inverse $f^{-\star}$. Note also that $(f \star g)^{-\star}=g^{-\star} \star f^{-\star}$ where it is defined. We refer the reader to [24, p. 125-129] for the details on the $\star$-product and $\star$-inverse.

The $\star$-product can be related to the pointwise product as described in the following result, [24, Proposition 4.3.22]:

Proposition 3.6. Let $\Omega \subseteq \mathbb{H}$ be an axially symmetric s-domain, $f, g: \Omega \rightarrow$ $\mathbb{H}$ be slice hyperholomorphic functions. Then

$$
(f \star g)(p)=f(p) g\left(f(p)^{-1} p f(p)\right)
$$

for all $p \in \Omega, f(p) \neq 0$, while $(f \star g)(p)=0$ when $p \in \Omega, f(p)=0$.

An immediate consequence is the following: 
Corollary 3.7. If $(f \star g)(p)=0$ then either $f(p)=0$ or $f(p) \neq 0$ and $g\left(f(p)^{-1} p f(p)\right)=0$.

Remark 3.8. Corollary 3.7 applies in particular to polynomials, allowing to recover a well known result, see [39]: if a polynomial $\mathcal{Q}(p)$ factors as

$$
\mathcal{Q}(p)=\left(p-\alpha_{1}\right) \star \ldots \star\left(p-\alpha_{n}\right), \quad \alpha_{j+1} \neq \bar{\alpha}_{j}, j=1, \ldots, n-1
$$

then $\alpha_{1}$ is a root of $\mathcal{Q}(p)$ while all the other zeroes $\tilde{\alpha}_{j}, j=2, \ldots, n$ belong to the spheres $\left[\alpha_{j}\right], j=2, \ldots, n$. The decomposition of the polynomial $\mathcal{Q}$, in general, is not unique.

Note that when $\alpha_{j+1}=\bar{\alpha}_{j}$ then $\mathcal{Q}(p)$ contains the second degree factor $p^{2}+2 \operatorname{Re}\left(\alpha_{j}\right) p+\left|\alpha_{j}\right|^{2}$ and the zero set of $\mathcal{Q}(p)$ contains the whole sphere $\left[\alpha_{j}\right]$. We will say that $\left[\alpha_{j}\right]$ is a spherical zero of the polynomial $\mathcal{Q}$.

Remark 3.9. Assume that $\mathcal{Q}(p)$ factors as in (3.4) and assume that $\alpha_{j} \in\left[\alpha_{1}\right]$ for all $j=2, \ldots, n$. Then the only root of $\mathcal{Q}(p)$ is $p=\alpha_{1}$, see [40, Lemma 2.2.11], [41, p. 519] the decomposition in linear factors is unique, and $\alpha_{1}$ is the only root of $\mathcal{Q}$.

Assume that $\left[\alpha_{j}\right]$ is a spherical zero. Then, for any $a_{j} \in\left[\alpha_{j}\right]$ we have

$$
p^{2}+2 \operatorname{Re}\left(\alpha_{j}\right) p+\left|\alpha_{j}\right|^{2}=\left(p-a_{j}\right) \star\left(p-\bar{a}_{j}\right)=\left(p-\bar{a}_{j}\right) \star\left(p-a_{j}\right)
$$

thus showing that both $a_{j}$ and $\bar{a}_{j}$ are zeroes of multiplicity 1 . So we can say that the (points of the) sphere $\left[\alpha_{j}\right]$ have multiplicity 1 . Thus the multiplicity of a spherical zero $\left[\alpha_{j}\right]$ equals the exponent of $p^{2}+2 \operatorname{Re}\left(\alpha_{j}\right) p+\left|\alpha_{j}\right|^{2}$ in a factorization of $\mathcal{Q}(p)$.

The discussion in the previous remark justifies the following:

Definition 3.10. Let

$$
\mathcal{Q}(p)=\left(p-\alpha_{1}\right) \star \ldots \star\left(p-\alpha_{n}\right), \quad \alpha_{j+1} \neq \bar{\alpha}_{j}, \quad j=1, \ldots, n-1 .
$$

We say that $\alpha_{1}$ is a zero of $\mathcal{Q}$ of multiplicity 1 if $\alpha_{j} \notin\left[\alpha_{1}\right]$ for $j=2, \ldots, n$. We say that $\alpha_{1}$ is a zero of $\mathcal{Q}$ of multiplicity $n \geq 2$ if $\alpha_{j} \in\left[\alpha_{1}\right]$ for all $j=2, \ldots, n$.

Assume now that $\mathcal{Q}(p)$ contains the factor $\left(p^{2}+2 \operatorname{Re}\left(\alpha_{j}\right) p+\left|\alpha_{j}\right|^{2}\right)$ and $\left[\alpha_{j}\right]$ is a zero of $\mathcal{Q}(p)$. We say that the multiplicity of the spherical zero $\left[\alpha_{j}\right]$ is $m_{j}$ if $m_{j}$ is the maximum of the integers $m$ such that $\left(p^{2}+2 \operatorname{Re}\left(\alpha_{j}\right) p+\left|\alpha_{j}\right|^{2}\right)^{m}$ divides $\mathcal{Q}(p)$.

Note that the notion of multiplicity of a spherical zero given in [34 is different since, under the same conditions described in Definition 3.10, it would be $2 m_{j}$.

Remark 3.11. The polynomial $\mathcal{Q}(p)$ can be factored as follows, see e.g. 34, Theorem 2.1]:

$$
\mathcal{Q}(p)=\prod_{j=1}^{r}\left(p^{2}+2 \operatorname{Re}\left(\alpha_{j}\right) p+\left|\alpha_{j}\right|^{2}\right)^{m_{j}}\left(\prod_{i=1}^{\star s} \prod_{j=1}^{\star n_{i}}\left(p-\alpha_{i j}\right)\right) a
$$


where $\prod^{\star}$ denotes the $\star$-product of the factors, $\left[\alpha_{i}\right] \neq\left[\alpha_{j}\right]$ for $i \neq j, \alpha_{i j} \in\left[a_{i}\right]$ for all $j=1, \ldots, n_{i}$ and $\left[a_{i}\right] \neq\left[a_{\ell}\right]$ for $i \neq \ell$. Note that

$$
\operatorname{deg}(\mathcal{Q})=\sum_{j=1}^{r} 2 m_{j}+\sum_{i=1}^{s} n_{i} .
$$

Definition 3.12. Let $a \in \mathbb{H},|a|<1$. The function

$$
B_{a}(p)=(1-p \bar{a})^{-\star} \star(a-p) \frac{\bar{a}}{|a|}
$$

is called a Blaschke factor at $a$.

Remark 3.13. Using Proposition 3.6, $B_{a}(p)$ can be rewritten as

$$
B_{a}(p)=(1-\tilde{p} \bar{a})^{-1}(a-\tilde{p}) \frac{\bar{a}}{|a|}
$$

where $\tilde{p}=(1-p a)^{-1} p(1-p a)$.

The following result is immediate, see [7]:

Proposition 3.14. Let $a \in \mathbb{H},|a|<1$. The Blaschke factor $B_{a}$ is a slice hyperholomorphic function in $\mathbb{B}$.

As one expects, $B_{a}(p)$ has only one zero at $p=a$ and analogously to what happens in the case of the zeroes of a function, the product of two Blaschke factors of the form $B_{a}(p) \star B_{\bar{a}}(p)$ gives the Blaschke factor with zeroes at the sphere $[a]$. Thus we give the following definition:

Definition 3.15. Let $a \in \mathbb{H},|a|<1$. The function

$$
B_{[a]}(p)=\left(1-2 \operatorname{Re}(a) p+p^{2}|a|^{2}\right)^{-1}\left(|a|^{2}-2 \operatorname{Re}(a) p+p^{2}\right)
$$

is called Blaschke factor at the sphere $[a]$.

Theorem 5.16 in [7] assigns a Blaschke product having zeroes at a given set of points $a_{j}$ with multiplicities $n_{j}, j \geq 1$ and at spheres $\left[c_{i}\right]$ with multiplicities $m_{i}, i \geq 1$, where the multiplicities are meant as exponents of the factors $\left(p-a_{j}\right)$ and $\left(p^{2}-\operatorname{Re}\left(a_{j}\right) p+\left|a_{j}\right|^{2}\right)$, respectively. In view of Definition 3.10, the polynomial $\left(p-a_{j}\right)^{\star n_{j}}$ is not the unique polynomial having a zero at $a_{j}$ with the given multiplicity $n_{j}$, thus the Blaschke product $\prod_{j=1}^{\star n_{j}} B_{a_{j}}$ is not the unique Blaschke product having zero at $a_{j}$ with multiplicity $n_{j}$.

We give below a form of Theorem 5.16 in [7] in which we use the notion of multiplicity in Definition 3.10 .

Theorem 3.16. A Blaschke product having zeroes at the set

$$
Z=\left\{\left(a_{1}, n_{1}\right), \ldots,\left(\left[c_{1}\right], m_{1}\right), \ldots\right\}
$$

where $a_{j} \in \mathbb{B}, a_{j}$ have respective multiplicities $n_{j} \geq 1, a_{j} \neq 0$ for $j=1,2, \ldots$, $\left[a_{i}\right] \neq\left[a_{j}\right]$ if $i \neq j, c_{i} \in \mathbb{B}$, the spheres $\left[c_{j}\right]$ have respective multiplicities 
$m_{j} \geq 1, j=1,2, \ldots,\left[c_{i}\right] \neq\left[c_{j}\right]$ if $i \neq j$ and

$$
\sum_{i, j \geq 1}\left(n_{i}\left(1-\left|a_{i}\right|\right)+2 m_{j}\left(1-\left|c_{j}\right|\right)\right)<\infty
$$

is of the form

$$
\prod_{i \geq 1}\left(B_{\left[c_{i}\right]}(p)\right)^{m_{i}} \prod_{i \geq 1}^{\star} \prod_{j=1}^{\star n_{i}}\left(B_{\alpha_{i j}}(p)\right)
$$

where $n_{j} \geq 1, \alpha_{11}=a_{1}$ and $\alpha_{i j}$ are suitable elements in $\left[a_{i}\right]$ for $j=2,3, \ldots$.

Proof. The fact that (3.7) ensure the convergence of the product follows from [7, Theorem 5.6]. The zeroes of the pointwise product $\prod_{i \geq 1}\left(B_{\left[c_{i}\right]}(p)\right)^{m_{i}}$ correspond to the given spheres with their multiplicities. Let us consider the product:

$$
\prod_{i=1}^{\star n_{1}}\left(B_{\alpha_{i 1}}(p)\right)=B_{\alpha_{11}}(p) \star B_{\alpha_{12}}(p) \star \ldots \star B_{\alpha_{1 n_{1}}}(p) .
$$

As we already observed in the proof of Proposition 5.10 in [7] this product admits a zero at the point $\alpha_{11}=a_{1}$ and it is a zero of multiplicity 1 if $n_{1}=1$; if $n_{1} \geq 2$, the other zeroes are $\tilde{\alpha}_{12}, \ldots, \tilde{\alpha}_{1 n_{1}}$ where $\tilde{\alpha}_{1 j}$ belong to the sphere $\left[\alpha_{1 j}\right]=\left[a_{1}\right]$. This fact can be seen directly using formula (3.3). Thus, according to Remark 3.8, $a_{1}$ is a zero of multiplicity $n_{1}$. Let us now consider $r \geq 2$ and

$$
\prod_{j=1}^{\star n_{r}}\left(B_{\alpha_{r j}}(p)\right)=B_{\alpha_{r 1}}(p) \star \ldots \star B_{\alpha_{r n_{r}}}(p),
$$

and set

$$
B_{r-1}(p):=\prod_{i \geq 1}^{\star(r-1)} \prod_{j=1}^{\star n_{i}}\left(B_{\alpha_{i j}}(p)\right)
$$

Then

$$
B_{r-1}(p) \star B_{\alpha_{r 1}}(p)=B_{r-1}(p) B_{\alpha_{r 1}}\left(B_{r-1}(p)^{-1} p B_{r-1}(p)\right)
$$

has a zero at $a_{r}$ if and only if $B_{\alpha_{r 1}}\left(B_{r-1}\left(a_{r}\right)^{-1} a_{r} B_{r-1}\left(a_{r}\right)\right)=0$, i.e. if and only if $\alpha_{r 1}=B_{r-1}\left(a_{r}\right)^{-1} a_{r} B_{r-1}\left(a_{r}\right)$. If $n_{r}=1$ then $a_{r}$ is a zero of multiplicity 1 while if $n_{r} \geq 2$, all the other zeroes of the product (3.8) belongs to the sphere $\left[a_{r}\right]$ thus, by Remark 3.8 , the zero $a_{r}$ has multiplicity $n_{r}$. This completes the proof.

Remark 3.17. In the case in which one has to construct a Blaschke product having a zero at $a_{i}$ with multiplicity $n_{i}$ by prescribing the factors $\left(p-a_{i 1}\right) \star$ $\cdots \star\left(p-a_{i n_{i}}\right), a_{i j} \in\left[a_{i}\right]$ for all $j=1, \ldots, n_{i}$, the factors in the Blaschke product must be chosen accordingly (see the proof of Theorem 3.16).

Proposition 3.18. The $\star$-inverse of $B_{a}$ and $B_{[a]}$ are $B_{\bar{a}^{-1}}, B_{\left[a^{-1}\right]}$ respectively. 
Proof. It follows from straightforward computations, by verifying that the products $B_{a} \star B_{\bar{a}^{-1}}$ and $B_{[a]} \star B_{\left[a^{-1}\right]}$ equal 1 .

Definition 3.19. A Blaschke product of the form

$$
B(p)=\prod_{i=1}^{r}\left(B_{\left[c_{i}\right]}(p)\right)^{m_{i}} \prod_{i=1}^{\star s} \prod_{j=1}^{\star n_{i}}\left(B_{\alpha_{i j}}(p)\right),
$$

is said to have degree $d=\sum_{i=1}^{r} 2 m_{i}+\sum_{j=1}^{s} n_{j}$.

Proposition 3.20. Let $B(p)$ be a Blaschke product as in (3.9). Then $\operatorname{dim}(\mathcal{H}(B))=\operatorname{deg} B$.

Proof. Let us rewrite $B(p)$ as

$$
B(p)=\prod_{i=1}^{r}\left(B_{c_{i}}(p) \star B_{\bar{c}_{i}}(p)\right)^{m_{i}} \prod_{i=1}^{\star s} \prod_{j=1}^{\star n_{i}}\left(B_{\alpha_{i j}}(p)\right)=\prod_{j=1}^{\star d} B_{\beta_{j}}(p),
$$

$d=\operatorname{deg} B$. Let us first observe that in the case in which the factors $B_{\beta_{j}}$ are such that no three of the quaternions $\beta_{j}$ belong to the same sphere, then the statement follows from the fact that $\mathcal{H}\left(B_{\beta_{j}}\right)$ is the span of $\left(1-p \bar{\beta}_{j}\right)^{-\star}$. MOreover $\left(1-p \bar{\beta}_{1}\right)^{-\star}, \ldots,\left(1-p \bar{\beta}_{d}\right)^{-\star}$ are linearly independent in the Hardy space $\mathbf{H}_{2}(\mathbb{B})$, see [4, Remark 3.1]. So we now assume that $d \geq 3$ and at least three among the $\beta_{j}$ 's belong the same sphere. We proceed by induction. Assume that $d=3$ and $\beta_{1}, \beta_{2}, \beta_{3}$ belong to the same sphere. Since

$$
\begin{aligned}
K_{B}(p, q) & =\sum_{n} p^{n}\left(1-B(p) B(q)^{*}\right) \bar{q}^{n}=\sum_{n} p^{n}\left(1-B_{\beta_{1}}(p) B_{\beta_{1}}(q)^{*}\right) \bar{q}^{n} \\
& +B_{\beta_{1}}(p) \star \sum_{n} p^{n}\left(1-B_{\beta_{2}}(p) B_{\beta_{2}}(q)^{*}\right) \bar{q}^{n} \star_{r} B_{\beta_{1}}(q)^{*} \\
& +B_{\beta_{1}}(p) \star B_{\beta_{2}}(p) \star \sum_{n} p^{n}\left(1-B_{\beta_{3}}(p) B_{\beta_{3}}(q)^{*}\right) \bar{q}^{n} \star_{r} B_{\beta_{1}}(q)^{*} \star_{r} B_{\beta_{1}}(q)^{*}
\end{aligned}
$$

we have

$$
\mathcal{H}\left(B_{\beta_{1}}\right)+B_{\beta_{1}} \star \mathcal{H}\left(B_{\beta_{2}}\right)+B_{\beta_{1}} \star B_{\beta_{2}} \star \mathcal{H}\left(B_{\beta_{3}}\right) .
$$

Now note that $\mathcal{H}\left(B_{\beta_{1}}\right)$ is spanned by $f_{1}(p)=\left(1-p \bar{\beta}_{1}\right)^{-\star}, B_{\beta_{1}} \star \mathcal{H}\left(B_{\beta_{2}}\right)$ is spanned by $f_{2}(p)=B_{\beta_{1}}(p) \star\left(1-p \bar{\beta}_{2}\right)^{-\star}$ and, finally, $B_{\beta_{1}} \star B_{\beta_{2}} \star \mathcal{H}\left(B_{\beta_{3}}\right)$ is spanned by $f_{3}(p)=B_{\beta_{1}}(p) \star B_{\beta_{2}}(p) \star\left(1-p \bar{\beta}_{3}\right)^{-\star}$. By using the reproducing property of $f_{1}$ we have $\left[f_{1}, f_{2}\right]=0$ and $\left[f_{1}, f_{3}\right]=0$ (here $[\cdot, \cdot]$ denotes the inner product in $\left.\mathbf{H}_{2}(\mathbb{B})\right)$. Observe that

$$
\left[f_{2}, f_{3}\right]=\left[\left(1-p \bar{\beta}_{2}\right)^{-\star}, B_{\beta_{2}}(p) \star\left(1-p \bar{\beta}_{3}\right)^{-\star}\right]=0
$$

since the left multiplication by $B_{\beta_{1}}(p)$ is an isometry in $\mathbf{H}_{2}(\mathbb{B})$ and by the reproducing property of $\left(1-p \bar{\beta}_{2}\right)^{-\star}$. So $f_{1}, f_{2}, f_{3}$ are orthogonal in $\mathbf{H}_{2}(\mathbb{B})$ and so they are linearly independent. We conclude that the sum (3.10) is direct and has dimension 3. Now assume that the assertion hold when $d=n$ and there in $B(p)$ are at least three Blaschke factors at points on the same 
sphere. We show that the assertion holds for $d=n+1$. We generalize the above discussion by considering

$$
\begin{aligned}
& \left(\mathcal{H}\left(B_{\beta_{1}}\right)+B_{\beta_{1}} \star \mathcal{H}\left(B_{\beta_{2}}\right)+\cdots+B_{\beta_{1}} \star \cdots \star B_{\beta_{n-1}} \star \mathcal{H}\left(B_{\beta_{n}}\right)+\cdots+\right. \\
& +B_{\beta_{1}} \star \cdots \star B_{\beta_{n}} \star \mathcal{H}\left(B_{\beta_{n+1}}\right) .
\end{aligned}
$$

Let us denote, as before, by $f_{1}(p)=\left(1-p \bar{\beta}_{1}\right)^{-\star}$ a generator of $\mathcal{H}\left(B_{\beta_{1}}\right)$ and by $f_{j}(p)=B_{\beta_{1}} \star \cdots \star B_{\beta_{j-1}} \star\left(1-p \bar{\beta}_{j}\right)^{-\star}$ a generator of $B_{\beta_{1}} \star \cdots \star B_{\beta_{j-1}} \star \mathcal{H}\left(B_{\beta_{j}}\right)$, $j=1, \ldots, n+1$. By the induction hypothesis, the sum of the first $n$ terms is direct so we show that $\left[f_{j}, f_{n+1}\right]=0$ for $j=1, \ldots, n$. This follows, as before, from the fact that the multiplication by a Blaschke factor is an isometry and by the reproducing property. The statement follows.

We now introduce the Blaschke factors in the half-space

$$
\mathbb{H}_{+}=\{p \in \mathbb{H}: \operatorname{Re}(p)>0\} .
$$

Definition 3.21. For $a \in \mathbb{H}_{+}$set

$$
b_{a}(p)=(p+\bar{a})^{-\star} \star(p-a) .
$$

The function $b_{a}(p)$ is called Blaschke factor at $a$ in the half space $\mathbb{H}_{+}$.

Remark 3.22. The function $b_{a}(p)$ is defined outside the sphere $[-a]$ and it has a zero at $p=a$. A Blaschke factor $b_{a}$ is slice hyperholomorphic in $\mathbb{H}^{+}$.

As before, we can also introduce Blaschke factors at spheres:

Definition 3.23. For $a \in \mathbb{H}_{+}$set

$$
b_{[a]}(p)=\left(p^{2}+2 \operatorname{Re}(a) p+|a|^{2}\right)^{-1}\left(p^{2}-2 \operatorname{Re}(a) p+|a|^{2}\right) .
$$

The function $b_{a}(p)$ is called Blaschke factor at the sphere $[a]$ in the half space $\mathbb{H}_{+}$.

We now state the following result whose proof mimics the lines of the proof of Theorem 3.16 with obvious changes. Note that an analog of Remark 3.17 holds also in this case.

Theorem 3.24. A Blaschke product having zeroes at the set

$$
Z=\left\{\left(a_{1}, n_{1}\right), \ldots,\left(\left[c_{1}\right], m_{1}\right), \ldots\right\}
$$

where $a_{j} \in \mathbb{H}_{+}$, $a_{j}$ have respective multiplicities $n_{j} \geq 1,\left[a_{i}\right] \neq\left[a_{j}\right]$ if $i \neq j$, $c_{i} \in \mathbb{H}_{+}$, the spheres $\left[c_{j}\right]$ have respective multiplicities $m_{j} \geq 1, j=1,2, \ldots$, $\left[c_{i}\right] \neq\left[c_{j}\right]$ if $i \neq j$ and

$$
\sum_{i, j \geq 1}\left(n_{i}\left(1-\left|a_{i}\right|\right)+2 m_{j}\left(1-\left|c_{j}\right|\right)\right)<\infty
$$

is given by

$$
\prod_{i \geq 1}\left(b_{\left[c_{i}\right]}(p)\right)^{m_{i}} \prod_{i \geq 1}^{\star} \prod_{j=1}^{\star n_{i}}\left(b_{\alpha_{i j}}(p)\right),
$$

where $\alpha_{11}=a_{1}$ and $\alpha_{i j}$ are suitable elements in $\left[a_{i}\right]$ for $i=2,3, \ldots$ 
Let $f(p)=\sum_{n=-\infty}^{+\infty}\left(p-p_{0}\right)^{\star n} a_{n}$ where $a_{n} \in \mathbb{H}$. Following the standard nomenclature and [45] we now give the definition of singularity of a slice regular function:

Definition 3.25. A function $f$ has a pole at the point $p_{0}$ if there exists $m \geq 0$ such that $a_{-k}=0$ for $k>m$. The minimum of such $m$ is called the order of the pole;

If $p$ is not a pole then we call it an essential singularity for $f$;

$f$ has a removable singularity at $p_{0}$ if it can be extended in a neighborhood of $p_{0}$ as a slice hyperholomorphic function.

A function $f$ has a pole at $p_{0}$ if and only if its restriction to a complex plane has a pole. In this framework there can be poles of order 0 . To give an example, let $I \in \mathbb{S}$; then the function $(p+I)^{-\star}=\left(p^{2}+1\right)^{-1}(p-I)$ has a pole of order 0 at the point $-I$ which, however, is not a removable singularity, see [24, p.55].

Definition 3.26. Let $\Omega$ be an axially symmetric s-domain in $\mathbb{H}$. We say that a function $f: \Omega \rightarrow \mathbb{H}$ is slice hypermeromorphic in $\Omega$ if $f$ is slice hyperholomorphic in $\Omega^{\prime} \subset \Omega$ such that every point in $\Omega \backslash \Omega^{\prime}$ is a pole.

\section{Some ReSUlts From QUATERnioniC FUnCTIONAL ANALYSIS}

The tools from quaternionic functional analysis needed in the present paper are of two kinds. On one hand, we need some results from the theory of quaternionic Pontryagin spaces, taken essentially from 14. On the other hand, we also need the quaternionic version of the Schauder-Tychonoff theorem in order to prove an invariant subspace theorem for contractions in Pontryagin spaces. More generally we note that in our on-going project on Schur analysis in the slice hyperholomorphic setting we were lead to prove a number of results in quaternionic functional analysis not readily available in the literature.

Operator theory in (quaternionic) Pontryagin spaces plays an important role in (quaternionic) Schur analysis, and we here recall some definitions and results needed in the sequel. We refer to [14] for more information.

Definition 4.1. Let $\mathcal{V}$ be a right quaternionic vector space. The map

$$
[\cdot, \cdot]: \mathcal{V} \times \mathcal{V} \longrightarrow \mathbb{H}
$$

is called an inner product if it is a (right) sesquilinear form:

$$
\left[v_{1} c_{1}, v_{2} c_{2}\right]=\overline{c_{2}}\left[v_{1}, v_{2}\right] c_{1}, \quad \forall v_{1}, v_{2} \in \mathcal{V}, \text { and } c_{1}, c_{2} \in \mathbb{H},
$$

which is Hermitian in the sense that:

$$
[v, w]=\overline{[w, v]}, \quad \forall v, w \in \mathcal{V} .
$$


A quaternionic inner product space $\mathcal{V}$ is called a Pontryagin space if it can be written as a direct and orthogonal sum

$$
\mathcal{V}=\mathcal{V}_{+}[\oplus] \mathcal{V}_{-}
$$

where $\left(\mathcal{V}_{+},[\cdot, \cdot]\right)$ is a Hilbert space, and $\left(\mathcal{V}_{-},-[\cdot, \cdot]\right)$ is a finite dimensional Hilbert space. As in the complex case, the space $\mathcal{V}$ endowed with the form

$$
\langle h, g\rangle=\left[h_{+}, g_{+}\right]-\left[h_{-}, g_{-}\right]
$$

where $h=h_{+}+h_{-}$and $g=g_{+}+g_{-}$are the decompositions of $f, g \in \mathcal{V}$ along (4.1), is a Hilbert space and the norms associated with the inner products (4.2) are equivalent, and hence define the same topology. The notions of adjoint and contraction are defined as in the complex case, and Theorem 2.1 still holds in the quaternionic setting:

Theorem 4.2. [8, Theorem 7.2] A densely defined contraction between quaternionic Pontryagin spaces of the same index has a unique contractive extension and its adjoint is also a contraction.

A key result used in the proof of the Krein-Langer factorization is the following invariant subspace theorem.

Theorem 4.3. [5, Theorem 4.6] A contraction in a quaternionic Pontryagin space has a unique maximal invariant negative subspace, and it is one-to-one on it.

The arguments there follow the ones given in the complex case in [29], and require in particular to prove first a quaternionic version of the SchauderTychonoff theorem, and an associated lemma. We recall these for completeness:

Lemma 4.4. [5, Lemma 4.4] Let $\mathcal{K}$ be a compact convex subset of a locally convex linear quaternionic space $\mathcal{V}$ and let $T: \mathcal{K} \rightarrow \mathcal{K}$ be continuous. If $\mathcal{K}$ contains at least two points, then there exists a proper closed convex subset $\mathcal{K}_{1} \subset \mathcal{K}$ such that $T\left(\mathcal{K}_{1}\right) \subseteq \mathcal{K}_{1}$.

Theorem 4.5 (Schauder-Tychonoff). [5, Theorem 4.5] A compact convex subset of a locally convex quaternionic linear space has the fixed point property.

\section{Generalized Schur functions and their Realizations}

The definition of negative squares makes sense in the quaternionic setting since an Hermitian quaternionic matrix $H$ is diagonalizable: it can be written as $H=U D U^{*}$, where $U$ is unitary and $D$ is unique and with real entries. The number of strictly negative eigenvalues of $H$ is exactly the number of strictly negative elements of $D$, see 48. The one-to-one correspondence between reproducing kernel Pontryagin spaces and functions with a finite number of negative squares, proved in the classical case by [43, 44], extends to the Pontryagin space setting, see [14]. 
We first recall a definition. A quaternionic matrix $J$ is called a signature matrix if it is both self-adjoint and unitary. The index of $J$ is the number of strictly negative eigenvalues of $J$, and the latter is well defined because of the spectral theorem for quaternionic matrices. See e.g. [48].

Definition 5.1. Let $\Omega$ be an axially symmetric s-domain contained in the unit ball, let $J_{1} \in \mathbb{H}^{s \times s}$ and $J_{2} \in \mathbb{H}^{r \times r}$ be two signature matrix of the same index, and let $S$ be a $\mathbb{H}^{r \times s}$-valued function, slice hyperholomorphic in $\Omega$. Then $S$ is called a generalized Schur function if the kernel

$$
K_{S}(p, q)=\sum_{n=0}^{\infty} p^{n}\left(J_{2}-S(p) J_{1} S(q)^{*}\right) \bar{q}^{n}
$$

has a finite number of negative squares, say $\kappa$, in $\Omega$. We set $\kappa=$ ind $S$ and call it the index of $S$.

We will denote by $\mathscr{S}_{\kappa}\left(J_{1}, J_{2}\right)$ the family of generalized Schur functions of index $\kappa$.

Lemma 5.2. In the notation of Definition 5.1, let $x_{0} \in \Omega \cap \mathbb{R}$. Let $b(p)=$ $\frac{p+x_{0}}{1+p x_{0}}$. Then the function $S \circ b$ is a generalized Schur function slice hyperholomorphic at the origin and with the same index as $S$.

Proof. First of all, we note that $\left(1+p x_{0}\right)^{-\star}=\left(1+p x_{0}\right)^{-1}$ since $x_{0} \in \mathbb{R}$, and that $\left(1+p x_{0}\right)^{-1}$ commute with $p+x_{0}$ thus the rational function $b(p)$ is well defined. The result then follows from the formula

$$
\begin{aligned}
& \sum_{n=0}^{\infty} p^{n}\left(J_{2}-S(b(p)) J_{1} S(b(q))^{*}\right) \bar{q}^{n}=\left(1-x_{0}^{2}\right) \times \\
& \left.\quad \times\left(1+p x_{0}\right)^{-1}\left(\sum_{n=0}^{\infty} b(p)^{n}\left(J_{2}-S(b(p)) J_{1} S(b(q))^{*}\right) \overline{b(q)}\right)^{n}\right)\left(1+\bar{q} x_{0}\right)^{-1} .
\end{aligned}
$$

To show the validity of (5.1) we use [5, Proposition 2.22] to compute the left hand side which gives

$$
\sum_{n=0}^{\infty} p^{n}\left(J_{2}-S(b(p)) J_{1} S(b(q))^{*}\right) \bar{q}^{n}=\left(J_{2}-S(b(p)) J_{1} S(b(q))^{*}\right) \star(1-p \bar{q})^{-\star},
$$

where the $\star$-product is the left one and it is computed with respect to $p$. The right hand side of (5.1) can be computed in a similar was and gives

$$
\begin{aligned}
& \left.\left(1-x_{0}^{2}\right)\left(1+p x_{0}\right)^{-1}\left(\sum_{n=0}^{\infty} b(p)^{n}\left(J_{2}-S(b(p)) J_{1} S(b(q))^{*}\right) \overline{b(q)}\right)^{n}\right)\left(1+\bar{q} x_{0}\right)^{-1} \\
& \left(1-x_{0}^{2}\right)\left(1+p x_{0}\right)^{-1}\left(J_{2}-S(b(p)) J_{1} S(b(q))^{*}\right) \star(1-b(p) \overline{b(q)})^{-\star}\left(1+\bar{q} x_{0}\right)^{-1} .
\end{aligned}
$$


We now note that

$$
(1-b(p) \overline{b(q)})^{-\star}=\left(1-\frac{p+x_{0}}{1+p x_{0}} \frac{\bar{q}+x_{0}}{1+\bar{q} x_{0}}\right)^{-\star}=\frac{1}{1-x_{0}^{2}}\left(1+p x_{0}\right)(1-p \bar{q})^{-\star}\left(1+\bar{q} x_{0}\right)
$$

and substituting this expression in (5.3), and using the property that

$$
\left(J_{2}-S(b(p)) J_{1} S(b(q))^{*}\right) \star\left(1+p x_{0}\right)=\left(1+p x_{0}\right)\left(J_{2}-S(b(p)) J_{1} S(b(q))^{*}\right)
$$

since $x_{0} \in \mathbb{R}$, we obtain

$$
\begin{aligned}
\left(1-x_{0}^{2}\right) & \left(1+p x_{0}\right)^{-1}\left(J_{2}-S(b(p)) J_{1} S(b(q))^{*}\right) \\
& \star \frac{1}{1-x_{0}^{2}}\left(1+p x_{0}\right)(1-p \bar{q})^{-\star}\left(1+\bar{q} x_{0}\right)\left(1+\bar{q} x_{0}\right)^{-1} \\
& \left(1+p x_{0}\right)^{-1}\left(1+p x_{0}\right)\left(J_{2}-S(b(p)) J_{1} S(b(q))^{*}\right)(1-p \bar{q})^{-\star} \\
& =\left(J_{2}-S(b(p)) J_{1} S(b(q))^{*}\right)(1-p \bar{q})^{-\star}
\end{aligned}
$$

and the statement follows.

The reproducing kernel Pontryagin space $\mathcal{P}(S)$ associated with a generalized Schur function $S$, namely the space with reproducing kernel $K_{S}$, is a right quaternionic vector space, with functions taking values in a two-sided quaternionic vector space. To present the counterpart of (2.2) with $\mathcal{P}(S)$ as a state space we first recall the following result, see [5, Proposition 2.22].

Proposition 5.3. Let $A$ be a bounded linear operator from a right-sided quaternionic Hilbert $\mathcal{P}$ space into itself, and let $C$ be a bounded linear operator from $\mathcal{P}$ into $\mathcal{C}$, where $\mathcal{C}$ is a two sided quaternionic Hilbert space. The slice hyperholomorphic extension of $C(I-x A)^{-1}, 1 / x \in \rho_{S}(A) \cap \mathbb{R}$, is

$$
(C-\bar{p} C A)\left(I-2 \operatorname{Re}(p) A+|p|^{2} A^{2}\right)^{-1} .
$$

We will use the notation

$$
C \star(I-p A)^{-\star} \stackrel{\text { def. }}{=}(C-\bar{p} C A)\left(I-2 \operatorname{Re}(p) A+|p|^{2} A^{2}\right)^{-1} .
$$

For the following result see [6, 7]. First two remarks: in the statement, an observable pair is defined, as in the complex case, by (2.3). Next, we denote by $M^{*}$ the adjoint of a quaternionic bounded linear operator from a Pontryagin space $\mathcal{P}_{1}$ into a Pontryagin space $\mathcal{P}_{2}$ :

$$
\left[M p_{1}, p_{2}\right]_{\mathcal{P}_{2}}=\left[p_{1}, M^{*} p_{2}\right]_{\mathcal{P}_{1}}, \quad p_{1} \in \mathcal{P}_{1} \quad \text { and } \quad p_{2} \in \mathcal{P}_{2} .
$$

Theorem 5.4. Let $J_{1} \in \mathbb{H}^{s \times s}$ and $J_{2} \in \mathbb{H}^{r \times r}$ be two signature matrix of the same index, and let $S$ be slice hyperholomorphic in a neighborhood of the origin. Then, $S$ is in $\mathscr{S}_{\kappa}\left(J_{1}, J_{2}\right)$ if and only if it can written in the form

$$
S(p)=D+p C \star\left(I_{\mathcal{P}}-p A\right)^{-\star} B,
$$


where $\mathcal{P}$ is a right quaternionic Pontryagin space of index $\kappa$, the pair $(C, A)$ is observable, and the operator matrix

$$
M=\left(\begin{array}{ll}
A & B \\
C & D
\end{array}\right): \mathcal{P} \oplus \mathbb{H}^{s} \longrightarrow \mathcal{P} \oplus \mathbb{H}^{r}
$$

satisfies

$$
\left(\begin{array}{ll}
A & B \\
C & D
\end{array}\right)\left(\begin{array}{cc}
I_{\mathcal{P}} & 0 \\
0 & J_{1}
\end{array}\right)\left(\begin{array}{cc}
A & B \\
C & D
\end{array}\right)^{*}=\left(\begin{array}{cc}
I_{\mathcal{P}} & 0 \\
0 & J_{2}
\end{array}\right)
$$

The space $\mathcal{P}$ can be chosen to be the reproducing kernel Pontryagin space $\mathcal{P}(S)$ with reproducing kernel $K_{S}(p, q)$. Then the coisometric colligation (5.6) is given by:

$$
\begin{aligned}
(A f)(p) & = \begin{cases}p^{-1}(f(p)-f(0)), & p \neq 0, \\
f_{1}, & p=0,\end{cases} \\
(B v)(p) & = \begin{cases}p^{-1}(S(p)-S(0)) v, & p \neq 0, \\
s_{1} v, & p=0,\end{cases} \\
C f & =f(0), \\
D v & =S(0) v,
\end{aligned}
$$

where $v \in \mathbb{H}^{s}, S(p)=\sum_{n=0}^{\infty} p^{n} s_{n}$ and $f \in \mathcal{P}$ with power series $f(p)=$ $\sum_{n=0}^{\infty} p^{n} f_{n}$ at the origin.

Assume now in the previous theorem that $r=s, J_{1}=J_{2}=J$, and that $\operatorname{dim} \mathcal{P}(S)$ is finite. Then, equation (5.7) is an equality in finite dimensional spaces (or as matrices) and the function $S$ is called $J$-unitary. The function $S$ is moreover rational and its McMillan degree, denoted by $\operatorname{deg} S$, is the dimension of the space $\mathcal{P}(S)$ (we refer to [6] for the notion of rational slicehyperholomorphic functions. Suffices here to say that the restriction of $S$ to the real axis is a $\mathbb{H}^{r \times r}$-valued rational function of a real variable).

The $\star$-factorization $S=S_{1} \star S_{2}$ of $S$ as a $\star$-product of two $\mathbb{H}^{r \times r}$-valued $J$ unitary functions is called minimal if $\operatorname{deg} S=\operatorname{deg} S_{1}+\operatorname{deg} S_{2}$. When $\kappa=0$, $S$ is a minimal product of elements of three types, called Blaschke-Potapov factors, and was first introduced by V. Potapov in [42] in the complex case. We give now a formal definition of the Blaschke-Potapov factors:

Definition 5.5. A $\mathbb{H}^{r \times r}$-valued Blaschke-Potapov factor of the first kind (resp, second kind) is defined as:

$$
B_{a}(p, P)=I_{r}+\left(B_{a}(p)-I_{r}\right) P
$$

where $|a|<1$ (resp. $|a|>1$ ) and $J, P \in \mathbb{H}^{r \times r}, J$ being a signature matrix, and $P$ a matrix such that $P^{2}=P$ and $J P \geq 0$.

A $\mathbb{H}^{r \times r}$-valued Blaschke-Potapov factor of the third kind is defined as:

$$
I_{r}-k u \star\left(p+w_{0}\right) \star\left(p-w_{0}\right)^{-\star} u^{*} J
$$


where $u \in \mathbb{H}^{r}$ is $J$-neutral (meaning $u J u^{*}=0$ ), $\left|w_{0}\right|=1$ and $k>0$.

Remark 5.6. In the setting of circuit theory, Blaschke-Potapov factors of the third kind are also called Brune sections, see e.g. [26], 3].

In the sequel, by Blaschke product we mean the product of BlaschkePotapov factors. Note that the inverse of a Blaschke-Potapov factor of the first kind is $B_{a}^{-\star}(p, P)=I_{r}+\left(B_{a}(p)^{-\star}-I_{r}\right) P$.

When $\kappa>0$ there need not exist minimal factorizations. We refer to [10, 11] for examples in the complex-valued case. On the other hand, still when $\kappa>0$ but for $J=I_{r}$, a special factorization exists, as a $\star$-quotient of two Blaschke products. This is a special case of the factorization of Krein-Langer. The following result plays a key role in the proof of this factorization. It is specific of the case $J_{1}=I_{s}$ and $J_{2}=I_{r}$, which allows us to use the fact that the adjoint of a contraction between quaternionic Pontryagin spaces of the same index is still a contraction.

Proposition 5.7. In the notation of Theorem 5.4, assume $J_{1}=I_{s}$ and $J_{2}=I_{r}$. Then the operator $A$ is a Pontryagin contraction.

Proof. Equation (5.7) expresses that the operator matrix $M$ (defined by (5.6) ) is a coisometry, and in particular a contraction, between Pontryagin spaces of same index. Its adjoint is a Pontryagin space contraction (see [14]) and we have

$$
\left(\begin{array}{cc}
A & B \\
C & D
\end{array}\right)^{*}\left(\begin{array}{cc}
I_{\mathcal{P}} & 0 \\
0 & I_{r}
\end{array}\right)\left(\begin{array}{cc}
A & B \\
C & D
\end{array}\right) \leq\left(\begin{array}{cc}
I_{\mathcal{P}} & 0 \\
0 & I_{s}
\end{array}\right)
$$

It follows from this inequality that

$$
A^{*} A+C^{*} C \leq I_{s} .
$$

Since the range of $C$ is inside the Hilbert space $\mathbb{H}^{r}$ we have that $A^{*}$ is a contraction from $\mathcal{P}$ into itself, and so is its adjoint $A=\left(A^{*}\right)^{*}$.

\section{The FACTORIZATION THEOREM}

Below we prove a version of the Krein-Langer factorization theorem in the slice hyperholomorphic setting which generalizes [8, Theorem 9.2]. The role of the Blaschke factors $B_{a}$ in the scalar case is played here by the Blaschke-Potapov factors with $J=I$.

Theorem 6.1. Let $J_{1}=I_{s}$ and $J_{2}=I_{r}$, and let $S$ be a $\mathbb{H}^{r \times s}$-valued generalized Schur function of index $\kappa$. Then there exists a $\mathbb{H}^{r \times r}$-valued Blaschke product $B_{0}$ of degree $\kappa$ and a $\mathbb{H}^{r \times s}$-valued Schur function $S_{0}$ such that

$$
S(p)=\left(B_{0}^{-\star} \star S_{0}\right)(p) .
$$

Proof. We proceed in a number of steps:

STEP 1: One can assume that $S$ is slice hyperholomorphic at the origin. 
To check this, we note that whenever $f=g \star h$, we have $f \circ b=(g \circ b) \star(h \circ b)$ where $b(p)=\frac{p+x_{0}}{1+p x_{0}}, x_{0} \in \mathbb{R}$. This equality is true on $\Omega \cap \mathbb{R}_{+}$, and extends to $\Omega$ by slice hyperholomorphic extension. Thus, taking into account Lemma 5.2. we now assume $0 \in \Omega$.

STEP 2: Let (5.5) be a coisometric realization of $S$. Then $A$ has a unique maximal strictly negative invariant subspace $\mathcal{M}$.

Indeed, $A$ is a contraction as proved in Proposition 5.7. The result then follows from Theorem 4.3 .

The rest of the proof is as in [8], and is as follows. Let $\mathcal{M}$ be the space defined in STEP 2, and let $A_{\mathcal{M}}, C_{\mathcal{M}}$ denote the matrix representations of $A$ and $C$, respectively, in a basis of $\mathcal{M}$, and let $G_{\mathcal{M}}$ be the corresponding Gram matrix. It follows from (5.9) that

$$
A_{\mathcal{M}}^{*} G_{\mathcal{M}} A_{\mathcal{M}} \leq G_{\mathcal{M}}-C_{\mathcal{M}}^{*} C_{\mathcal{M}}
$$

STEP 3: The equation

$$
A_{\mathcal{M}}^{*} P_{\mathcal{M}} A_{\mathcal{M}}=P_{\mathcal{M}}-C_{\mathcal{M}}^{*} C_{\mathcal{M}}
$$

has a unique solution. It is strictly negative and $\mathcal{M}$ endowed with this metric is contractively included in $\mathcal{P}(S)$.

Recall that the S-spectrum of an operator $A$ is defined as the set of quaternions $p$ such that $A^{2}-2 \operatorname{Re}(p) A+|p|^{2} I$ is not invertible, see [24]. Then, the first two claims follow from the fact that the S-spectrum of $A_{\mathcal{M}}$, which coincides with the right spectrum of $A_{\mathcal{M}}$, is outside the closed unit ball. Moreover, the matrix $G_{\mathcal{M}}-P_{\mathcal{M}}$ satisfies

$$
A_{\mathcal{M}}^{*}\left(G_{\mathcal{M}}-P_{\mathcal{M}}\right) A_{\mathcal{M}} \leq G_{\mathcal{M}}-P_{\mathcal{M}},
$$

or equivalently (since $A$ is invertible)

$$
G_{\mathcal{M}}-P_{\mathcal{M}} \leq A_{\mathcal{M}}^{-*}\left(G_{\mathcal{M}}-P_{\mathcal{M}}\right) A_{\mathcal{M}}^{-1}
$$

and so, for every $n \in \mathbb{N}$,

$$
G_{\mathcal{M}}-P_{\mathcal{M}} \leq\left(A_{\mathcal{M}}^{-*}\right)^{n}\left(G_{\mathcal{M}}-P_{\mathcal{M}}\right) A_{\mathcal{M}}^{-n}
$$

By the spectral theorem (see [23, Theorem 3.10, p. 616] and [24, Theorem 4.12 .6$, p. 155] for the spectral radius theorem) we have:

$$
\lim _{n \rightarrow \infty}\left\|A_{\mathcal{M}}^{-n}\right\|^{1 / n}=0
$$

and so $\lim _{n \rightarrow \infty}\left\|\left(A_{\mathcal{M}}^{-*}\right)^{n}\left(P_{\mathcal{M}}-G_{\mathcal{M}}\right) A_{\mathcal{M}}^{-n}\right\|=0$. Thus entry-wise

$$
\lim _{n \rightarrow \infty}\left(A_{\mathcal{M}}^{-*}\right)^{n}\left(P_{\mathcal{M}}-G_{\mathcal{M}}\right) A_{\mathcal{M}}^{-n}=0
$$

and it follows from (6.1) that $G_{\mathcal{M}}-P_{\mathcal{M}} \leq 0$.

By [8, Proposition 8.8]

$$
\mathcal{M}=\mathcal{P}(B)
$$


when $\mathcal{M}$ is endowed with the $P_{\mathcal{M}}$ metric. Furthermore:

STEP 4: The kernel $K_{S}-K_{B}$ is positive.

Let $k_{\mathcal{M}}$ denote the reproducing kernel of $\mathcal{M}$ when endowed with the $\mathcal{P}(S)$ metric. Then

$$
k_{\mathcal{M}}(p, q)-K_{B}(p, q) \geq 0
$$

and

$$
K_{S}(p, q)-k_{\mathcal{M}}(p, q) \geq 0 .
$$

Moreover

$$
K_{S}(p, q)-K_{B}(p, q)=K_{S}(p, q)-k_{\mathcal{M}}(p, q)+k_{\mathcal{M}}(p, q)-K_{B}(p, q)
$$

and so it is positive definite.

Finally we apply [8, Proposition 5.1] to

$$
K_{S}(p, q)-K_{B}(p, q)=B(p) \star\left(I_{r}-S_{0}(p) S_{0}(q)^{*}\right) \star_{r} B(q)^{*}
$$

where $S_{0}(p)=B(p)^{-\star} \star S(p)$, to conclude that $S_{0}$ is a Schur function.

\section{The CASE OF THE HALF-SPACE}

Since the map (where $x_{0} \in \mathbb{R}_{+}$)

$$
p \mapsto\left(p-x_{0}\right)\left(p+x_{0}\right)^{-1}
$$

sends the right half-space onto the open unit ball, one can translate the previous results to the case of the half-space $\mathbb{H}_{+}$. In particular the BlaschkePotapov factors are of the form

$$
B_{a}(p, P)=I_{r}+\left(b_{a}(p)-1\right) P
$$

where $P$ is a matrix such that $P^{2}=P$ and $J P \geq 0$ where, in general, $J$ is signature matrix, and $a \in \mathbb{H}^{+}$. The factors of the third type are now functions of the form

$$
I_{r}-k u \star\left(p+w_{0}\right)^{-\star} u^{*} J
$$

where $u \in \mathbb{H}^{r}$ is such that $u J u^{*}=0$, and $w_{0}+\overline{w_{0}}=0, k>0$. The various definitions and considerations on rational $J$-unitary functions introduced in Section 5 have counterparts here. We will not explicit them, but restrict ourselves to the case $J_{1}=I_{s}$ and $J_{2}=I_{r}$, and only mention the counterpart of the Krein-Langer factorization in the half-space setting. We outline the results and leave the proofs to the reader.

In the setting of slice hyperholomorphic functions in $\mathbb{H}_{+}$the counterpart of the kernel $\sum_{n=0}^{\infty} p^{n} \bar{q}^{n}$ is

$$
k(p, q)=(\bar{p}+\bar{q})\left(|p|^{2}+2 \operatorname{Re}(p) \bar{q}+\bar{q}^{2}\right)^{-1} .
$$


Definition 7.1. The $\mathbb{H}^{r \times s}$-valued function $S$ slice hypermeromorphic in an axially symmetric s-domain $\Omega$ which intersects the positive real line belongs to the class $\mathscr{S}_{\kappa}\left(\mathbb{H}_{+}\right)$if the kernel

$$
K_{S}(p, q)=I_{r} k(p, q)-S(p) \star k(p, q) \star_{r} S(q)^{*}
$$

has $\kappa$ negative squares in $\Omega$, where $k(p, q)$ is defined in (7.1).

The following realization theorem has been proved in [5, Theorem 6.2].

Theorem 7.2. Let $x_{0}$ be a strictly positive real number. A $\mathbb{H}^{r \times s}$-valued function $S$ slice hyperholomorphic in an axially symmetric s-domain $\Omega$ containing $x_{0}$ is the restriction to $\Omega$ of an element of $\mathcal{S}_{\kappa}\left(\mathbb{H}_{+}\right)$if and only if it can be written as

$$
\begin{aligned}
S(p)=H & -\left(p-x_{0}\right)\left(G-\left(\bar{p}-x_{0}\right)\left(\bar{p}+x_{0}\right)^{-1} G A\right) \times \\
& \times\left(\frac{\left|p-x_{0}\right|^{2}}{\left|p+x_{0}\right|^{2}} A^{2}-2 \operatorname{Re}\left(\frac{p-x_{0}}{p+x_{0}}\right) A+I\right)^{-1} F,
\end{aligned}
$$

where $A$ is a linear bounded operator in a right-sided quaternionic Pontryagin space $\Pi_{\kappa}$ of index $\kappa$, and, with $B=-\left(I+x_{0} A\right)$, the operator matrix

$$
\left(\begin{array}{ll}
B & F \\
G & H
\end{array}\right):\left(\begin{array}{l}
\Pi_{k} \\
\mathbb{H}^{s}
\end{array}\right) \longrightarrow\left(\begin{array}{l}
\Pi_{k} \\
\mathbb{H}^{r}
\end{array}\right)
$$

is co-isometric. In particular $S$ has a unique slice hypermeromorphic extension to $\mathbb{H}_{+}$. Furthermore, when the pair $(G, A)$ is observable, the realization is unique up to a unitary isomorphism of Pontryagin right quaternionic spaces.

By an abuse of notation, we write

$$
S(p)=H-\left(p-x_{0}\right) G \star\left(\left(x_{0}+p\right) I+\left(p-x_{0}\right) B\right)^{-\star} F
$$

rather than (7.2).

In the following statement, the degree of the Blaschke product $B_{0}$ is the dimension of the associated reproducing kernel Hilbert space with reproducing kernel $K_{B_{0}}$.

Theorem 7.3. Let $S$ be a $\mathbb{H}^{r \times s}$-valued function slice hypermeromorphic in an axially symmetric s-domain $\Omega$ which intersects the positive real line. Then, $S \in \mathscr{S}_{\kappa}\left(\mathbb{H}_{+}\right)$if and only if it can be written as $S=B_{0}^{-\star} \star S_{0}$, where $B_{0}$ is a $\mathbb{H}^{r \times r}$-valued finite Blaschke product of degree $\kappa$, and $S_{0} \in \mathscr{S}_{0}\left(\mathbb{H}_{+}\right)$.

\section{REFERENCES}

[1] J. Agler. On the representation of certain holomorphic functions defined on a polydisk, volume 48 of Operator Theory: Advances and Applications, pages 47-66. Birkhäuser Verlag, Basel, 1990.

[2] D. Alpay. The Schur algorithm, reproducing kernel spaces and system theory. American Mathematical Society, Providence, RI, 2001. Translated from the 1998 French original by Stephen S. Wilson, Panoramas et Synthèses. 
[3] D. Alpay, V. Bolotnikov, P. Dewilde, A. Dijksma. Brune sections in the nonstationary case. Linear Algebra Appl., 343/344, 389-418, 2002.

[4] D. Alpay, V. Bolotnikov, F. Colombo, and I. Sabadini. Self-mappings of the quaternionic unit ball: multiplier properties, Schwarz-Pick inequality, and Nevanlinna-Pick interpolation problem. to appear Indiana Univ. Math. J. (2014).

[5] D. Alpay, F. Colombo, I. Lewkowicz, and I. Sabadini. Realizations of slice hyperholomorphic generalized contractive and positive functions. ArXiv e-prints, October 2013.

[6] D. Alpay, F. Colombo, and I. Sabadini. Schur functions and their realizations in the slice hyperholomorphic setting. Integral Equations and Operator Theory, 72:253-289, 2012.

[7] D. Alpay, F. Colombo, and I. Sabadini. Pontryagin-de Branges-Rovnyak spaces of slice hyperholomorphic functions. J. Anal. Math., 121:87-125, 2013.

[8] D. Alpay, F. Colombo, and I. Sabadini. Krein-Langer factorization and related topics in the slice hyperholomorphic setting. J. Geom. Anal. 24(2): 843-872, 2014.

[9] D. Alpay, A. Dijksma, J. Rovnyak, and H. de Snoo. Schur functions, operator colligations, and reproducing kernel Pontryagin spaces, volume 96 of Operator theory: Advances and Applications. Birkhäuser Verlag, Basel, 1997.

[10] D. Alpay and H. Dym. On applications of reproducing kernel spaces to the Schur algorithm and rational $J$-unitary factorization. In I. Gohberg, editor, I. Schur methods in operator theory and signal processing, volume 18 of Operator Theory: Advances and Applications, pages 89-159. Birkhäuser Verlag, Basel, 1986.

[11] D. Alpay and I. Gohberg. Unitary rational matrix functions. In I. Gohberg, editor, Topics in interpolation theory of rational matrix-valued functions, volume 33 of $\mathrm{Op}$ erator Theory: Advances and Applications, pages 175-222. Birkhäuser Verlag, Basel, 1988.

[12] D. Alpay, M. E. Luna Elizarraras, M. Shapiro, and D.C. Struppa. Basics of functional analysis with bicomplex scalars, and bicomplex Schur analysis. Springer Briefs in Mathematics, Springer, 2014.

[13] D. Alpay, B. Schneider, M. Shapiro, and D. Volok. Fonctions rationnelles et théorie de la réalisation: le cas hyper-analytique. Comptes Rendus Mathématiques, 336:975980, 2003.

[14] D. Alpay and M. Shapiro. Reproducing kernel quaternionic Pontryagin spaces. Integral Equations and Operator Theory, 50:431-476, 2004.

[15] D. Alpay and V. Vinnikov. Finite dimensional de Branges spaces on Riemann surfaces. J. Funct. Anal., 189(2):283-324, 2002.

[16] N. Aronszajn. Theory of reproducing kernels. Trans. Amer. Math. Soc., 68:337-404, 1950.

[17] T. Ya. Azizov and I.S. Iohvidov. Foundations of the theory of linear operators in spaces with indefinite metric. Nauka, Moscow, 1986. (Russian). English translation: Linear operators in spaces with an indefinite metric. John Wiley, New York, 1989.

[18] J. Ball, T. Trent, and V. Vinnikov. Interpolation and commutant lifting for multipliers on reproducing kernel Hilbert spaces. In Proceedings of Conference in honor of the 60-th birthday of M.A. Kaashoek, volume 122 of Operator Theory: Advances and Applications, pages 89-138. Birkhauser, 2001.

[19] A. Bloch. Les fonctions holomorphes et méromorphes dans le cercle-unité. Mémorial des sciences mathématiques, pages 1-61, 1926. Fascicule 20.

[20] J. Bognár. Indefinite inner product spaces. Ergebnisse der Mathematik und ihrer Grenzgebiete, Band 78. Springer-Verlag, Berlin, 1974.

[21] V. Bolotnikov and L. Rodman. Krein-Langer factorizations via pole triples. Integral Equations and Operator Theory, 47(2):169-195, 2003.

[22] C. Chamfy. Fonctions méromorphes sur le cercle unité et leurs séries de Taylor. Ann. Inst. Fourier, 8:211-251, 1958. 
[23] F. Colombo and I. Sabadini. On some properties of the quaternionic functional calculus. J. Geom. Anal., 19(3):601-627, 2009.

[24] F. Colombo, I. Sabadini, and D. C. Struppa. Noncommutative functional calculus. Theory and applications of slice hyperholomorphic functions, volume 289 of Progress in Mathematics. Birkhäuser/Springer Basel AG, Basel, 2011.

[25] P. Delsarte, Y. Genin, and Y. Kamp. Pseudo-Carathéodory functions and Hermitian Toeplitz matrices. Philips J. Res., 41(1):1-54, 1986.

[26] P. Dewilde and H. Dym. Lossless inverse scattering, digital filters, and estimation theory. IEEE Trans. Inform. Theory, 30 (4): 644-662, 1984.

[27] B. Dickinson, Ph. Delsarte, Y. Genin, and Y. Kamp. Minimal realizations of pseudopositive and pseudo-bounded rational matrices. IEEE Transactions on Circuits and Systems, 32:603-605, 1985.

[28] W.F. Donoghue. Monotone matrix functions and analytic continuation, volume 207 of Die Grundlehren der mathematischen Wissenschaften. Springer-Verlag, 1974.

[29] M. A. Dritschel and J. Rovnyak. Extension theorems for contractions on Kreı̌n spaces, volume 47 of Operator theory: Advances and Applications, pages 221-305. Birkhäuser Verlag, Basel, 1990.

[30] M. A. Dritschel and J. Rovnyak. Operators on indefinite inner product spaces. In P. Lancaster, editor, Lectures on operator theory and its applications (Waterloo, ON, 1994), volume 3 of Fields Inst. Monogr., pages 141-232. Amer. Math. Soc., Providence, RI, 1996.

[31] J. Dufresnoy. Le problème des coefficients pour certaines fonctions méromorphes dans le cercle unité. Ann. Acad. Sci. Fenn. Ser. A. I, no., 250/9:7, 1958.

[32] B. Fritzsche and B. Kirstein, editors. Ausgewählte Arbeiten zu den Ursprüngen der Schur-Analysis, volume 16 of Teubner-Archiv zur Mathematik. B.G. Teubner Verlagsgesellschaft, Stuttgart-Leipzig, 1991.

[33] G. Gentili, D. C. Struppa, A new theory of regular functions of a quaternionic variable, Advances in Mathematics, 216 (2007), 279-301.

[34] G. Gentili and D. C. Struppa. On the multiplicity of zeroes of polynomials with quaternionic coefficients. Milan J. Math., 76:15-25, 2008.

[35] I.S. Iohvidov, M.G. Krĕn, and H. Langer. Introduction to the spectral theory of operators in spaces with an indefinite metric. Akademie-Verlag, Berlin, 1982.

[36] M.G. Kreŭn and H. Langer. Über die verallgemeinerten Resolventen und die charakteristische Funktion eines isometrischen Operators im Raume $\Pi_{k}$. In Hilbert space operators and operator algebras (Proc. Int. Conf. Tihany, 1970), pages 353-399. North-Holland, Amsterdam, 1972. Colloquia Math. Soc. János Bolyai.

[37] M.G. Krĕn and H. Langer. Über einige Fortsetzungsprobleme, die eng mit der Theorie hermitescher Operatoren im Raume $\pi_{k}$ zusammenhangen. I. Einige Funktionenklassen und ihre Darstellungen. Math. Nachrichten, 77:187-236, 1977.

[38] M.G. Kreı̆n and H. Langer. Some propositions on analytic matrix functions related to the theory of operators in the space $\pi_{k}$. Acta Sci. Math., 43:181-205, 1981.

[39] T. Y. Lam. A first course in noncommutative rings, volume 131 of Graduate Texts in Mathematics. Springer-Verlag, New York, second edition, 2001.

[40] R. Pereira. Quaternionic Polynomials and Behavioral Systems. PhD thesis, University of Aveiro, 2006.

[41] R. Pereira and P. Vettori. Stability of quaternionic linear systems. IEEE Trans. Automat. Control, 51(3):518-523, 2006.

[42] V. P. Potapov. The multiplicative structure of $J$-contractive matrix functions. Amer. Math. Soc. Transl., 15: 131-243, 1960.

[43] L. Schwartz. Sous espaces hilbertiens d'espaces vectoriels topologiques et noyaux associés (noyaux reproduisants). J. Analyse Math., 13:115-256, 1964.

[44] P. Sorjonen. Pontryagin Raüme mit einem reproduzierenden Kern. Ann. Acad. Fenn. Ser. A, 1:1-30, 1973. 
[45] C. Stoppato. Singularities of slice regular functions. Math. Nachr., 285(10):1274-1293, 2012.

[46] T. Takagi. On an algebraic problem related to an analytic theorem of Carathéodory and Fejér and on an allied theorem of Landau. Japanese journal of mathematics, 1:83-93, 1924.

[47] T. Takagi. Remarks on an algebraic problem. Japanese journal of mathematics, 2:1317, 1925.

[48] F. Zhang. Quaternions and matrices of quaternions. Linear Algebra Appl., 251:21-57, 1997.

(DA) Department of Mathematics, Ben-Gurion University of the Negev, BEER-SHEVA 84105 ISRAEL

E-mail address: dany@math.bgu.ac.il

(FC) Politecnico di Milano, Dipartimento di Matematica, Via E. Bonardi, 9, 20133 Milano, Italy

E-mail address: fabrizio.colombo@polimi.it

(IS) Politecnico di Milano, Dipartimento di Matematica, Via E. Bonardi, 9, 20133 Milano, Italy

E-mail address: irene.sabadini@polimi.it 